\section{HOMOPHONIC SUBSTITUTION}

VAldemar C. RochaJr.

Departamento de Eletrônica e Sistemas, UFPE, Recife, PE, Brazil.

Presented by Cid B. DEAraúJo

Historically most of the secret-key cryptographic systems that have been broken were broken by exploiting the deviation of the statistics of the clear-text from that of a completely random sequence. Homophonic substitution is a venerable technique for converting a clear-text sequence into a random sequence. In 1988 Günther introduced an important generalization of homophonic substitution called variable-length homophonic substitution.

The purpose of this presentation is first to review the information-theoretic treatment of Günther's homophonic substitution and then show how to implement it with a finite memory, considering clear-text symbol probabilities which are rational numbers. Shannon's concept of a strongly-ideal cipher system will be reviewed in order to provide the motivation for using any kind of homophonic substitution. The precise definition of variable-length homophonic substitution is presented together with the necessary and sufficient condition for such a substitution to be perfect, i.e., to create a completely random sequence. By employing binary coding, perfect homophonic substitution can be achieved with the introduction of less than 2 bits of entropy in each encoded source letter, and can be implemented using less than 4 random bits per coded letter. Some properties of the geometric series, resulting from the base 2 expansion of the clear-text symbol probabilities, are presented and are used to establish an accurate lower bound for the redundancy in homophonic substitution. - (May 24, 2002 ).

\section{POLYANILINE-SILICON HETEROJUNCTIONS AS A SENSING DEVICE}

\section{Walter M. Azevedo}

Departamento de Química Fundamental, CCEN, Universidade Federal de Pernambuco, Cidade Universitária, 50670-901 Recife, PE, Brazil.

Presented by Gilberto F. DeSÁ

Increasing concern with environmental and personal protection together with widespread requirements for more accurate process control has created a need for new or improved sensors for measuring both physical and chemical parameters. This need for better sensors is strongly influenced by the increasing use of intelligent microelectronics for monitoring and control.

Among several sensing devices developed for a wide range of gases, from organic or inorganic pollutants which must be measured at parts per millions level or lower, the conducting polymer or more specifically polyaniline have been considered as a prominent new materials for the development of chemical sensors. The very great interest for these polymers is the relative ease of syntheses by chemical or electrochemical oxidative polymerizations of the monomers, and by the fact that the $\pi$-conjugated polymer behaves as a synthetic metal when simultaneously is in the doped and oxidized state. The combination of these two doping processes allows one to develop a material with interesting electronic, electrochemical, magnetic or optical properties.

In the last decade our group has been concentrated with the development of new synthetic routes to prepare conducting polymer to improve its solubility and processability, also we have been involved with the development of sensor for glucose (1-2), ammonia (3), salinity measurements (4), radiation detection (5) using polyaniline as a active support. In this meeting we will present the recent development of semiconductor-polymer heterojunction to be applied as a gamma radiation detection and as a gassensing device. - (May 24, 2002 ).

\section{NATURAL RADIOACTIVITY: OCCURRENCE AND AP- PLICATIONS ON ENVIRONMENTAL STUDIES}

José M. GodoY

IRD/CNEN/MCT and PUC-Rio, Rio de Janeiro, RJ, Brazil.

Presented by Gilberto F. DESÁ

Although the radioactivity concept has been intrinsically bounded to its weapon applications or to nuclear disasters, as that of Chernobyl, we should not forget that radioactivity is a natural phenomenon and there are radioactive substances in the air we breathe, in the food we eat and even in our clothes.

Natural radionuclides cover a large variety of chemical elements that allows several applications on environmental studies. One of the most known is dating, not only with ${ }^{14} \mathrm{C}$ but, also, with other radionuclides. The so-called radioactive disequilibrium of the uranium and the thorium series could be applied on the study of geochemical processes as water mixing, residence times and siltation rates.

The present talk aims, initially, to refresh the concept of radioactivity as natural phenomena, including natural 